\title{
Long-Term Trends in the Stroke Prognosis Among Rural Residents in China: A Population-Based Surveillance Study
}

\author{
Jing $\operatorname{Han}^{1, *}$ \\ Jie Liu $\mathbb{D D}^{1-4, *}$ \\ Yakun $\mathrm{Wu}^{1,5, *}$ \\ Qiuxing Lin ${ }^{1-3,5}$ \\ Dandan Guo (ID) \\ Xin Zhang' \\ Jun $T u^{1-3,5}$ \\ Xianjia Ning ${ }^{1-3,5}$ \\ Jinghua Wang $\mathbb{D}^{1-3,5}$
}

'Department of Neurology, Tianjin Medical University General Hospital, Tianjin, 300052, People's Republic of China; ' 2 Laboratory of Epidemiology, Tianjin Neurological Institute, Tianjin, 300052, People's Republic of China; ${ }^{3}$ Tianjin Neurological Institute, Key Laboratory of Post-Neuroinjury NeuroRepair and Regeneration in Central Nervous System, Ministry of Education and Tianjin City, Tianjin, 300052, People's Republic of China; ${ }^{4}$ Center of Clinical Epidemiology \& Evidence-Based Medicine, The Jizhou People's Hospital, Tianjin, 301999, People's Republic of China; ${ }^{5}$ Department of Neurology, Tangshan Gongren Hospital, Tangshan, 063000, People's Republic of China

*These authors contributed equally to this work

\begin{abstract}
Objective: Although stroke mortality has remained stable over the past decade, the long-term trends of stroke burden are scarce in China, especially in low-income populations. Thus, we aimed to explore the secular trends in the prognosis of stroke in a low-income population in China.

Methods: This was a population-based stroke surveillance study that included all of the participants in the Tianjin Brain Study. Stroke management and outcomes were evaluated for all stroke patients. Trends in the burden of stroke were assessed as annual percentage changes.
\end{abstract}

Results: Overall, 1462 stroke cases occurred from 1992 to 2018, and 58.7\% of patients were male. The rates of diagnosis by neuroimaging and of hospitalization increased noticeably across sexes and ages for all stroke types, and the greatest increases were observed among elderly women; diagnosis by neuroimaging increased by $4.7 \%$ and the rate of hospitalization increased by $12.4 \%$ (all $\mathrm{P}<0.001$ ). Stroke fatality decreased by $3.7 \%$ annually among those aged $<65$ years and by $3.1 \%$ among patients aged $\geq 65$ years; the corresponding rates were $7.4 \%$ and $3.5 \%$, respectively, for women. The recurrence rate of ischemic stroke significantly decreased among all men, by $9.6 \%$ for those aged $<65$ years and by $3.1 \%$ for those aged $\geq 65$ years; however, for women, this rate only significantly decreased for those aged $\geq 65$ years.

Conclusion: Our findings suggest that the allocation of medical resources has improved over the past decades. However, it is crucial to enhance secondary stroke prevention for women aged $<65$ years by controlling risk factors in low-income populations.

Keywords: stroke burden, case fatality, recurrence, trends, low-income population

\section{Introduction}

Stroke is the leading cause of death worldwide and has the highest disabilityadjusted life-years lost of any disease; over 2 million new cases are diagnosed annually in China. ${ }^{1-4}$ A global study pointed out that from 1990 to 2016, East Asia, especially China, had the highest age-standardized incidence of stroke at 354 per 100,000 person-years, followed by Eastern Europe, with an incidence of 181218 per 100,000 person-years. The lowest incidence is in central Latin America, 97 per 100,000 person-years. ${ }^{5}$ Previous studies have shown that, while stroke prevalence and incidence have increased markedly, mortality has remained stable over the past decade.$^{6-8}$ The National Epidemiological Survey of Stroke in China, which studied 480,687 individuals aged 20 years and older from 31 provinces between 2012 and 2013, reported an age-standardized stroke mortality of 115 cases per $100000 .^{7}$
Correspondence: Jinghua Wang;

Xianjia Ning

Email jwang3@tmu.edu.cn;

xning@tmu.edu.cn 
Nevertheless, stroke care and in-hospital outcomes have substantially improved following the reform of the national healthcare system in China. ${ }^{7}$ The most noticeable progress has been in the secondary prevention of stroke. The rates of recurrence within 1 year and case fatality both noticeably decreased from 2007 to 2012 , from $17.7 \%$ to $6.7 \%$ for recurrence and from $14.3 \%$ to $8.5 \%$ for case fatality. ${ }^{9-11}$ The Chinese government conducted a series of large-scale regional and national studies to evaluate the efficiency of secondary stroke prevention. ${ }^{12-14}$ However, long-term trends in stroke burden, as defined by stroke management and outcomes, remain scarce in China, notably among low-income populations.

Thus, we aimed to explore the secular trends in the prognosis of stroke in a low-income population in China from 1992 to 2018.

\section{Methods}

\section{Tianjin Brain Study}

The Tianjin Brain Study (TBS) is a population-based stroke surveillance study that has been ongoing in a township in Tianjin, China since 1985. Data collection for the TBS has been described in detail elsewhere. ${ }^{15-18}$ All participants in the TBS lived in the Yangjinzhuang township of Ji county in Tianjin, China. From 1992 to 1998, the permanent population of the area remained stable, between 14,583-14,936. All stroke events and allcause deaths were registered in real time, and the demographic changes were recorded annually.

The study protocol was approved by the ethics committee of Tianjin Medical University General Hospital (TMUGH), and written informed consent was obtained from each participant (contract number: IRB2018-099-01).

\section{Study Population and Design}

The current study was a population-based stroke surveillance study that included all of the participants in the TBS. Briefly, the study participants resided in 18 administrative villages in Yangjinzhuang, a township in Tianjin; 95\% of the residents were low-income farmers. The primary source of income was grain production, and the annual per capita income was $<100$ USD in 1991 and $<1000$ USD in $2010 .{ }^{19}$

\section{Definition of Stroke Type}

First-ever strokes were defined as the first occurrences (ie, there was no history of stroke in the patient's medical records) of rapidly developing signs of focal neurologic disturbances of presumed vascular etiology lasting $>24$ hours. ${ }^{20}$ Stroke events included the ischemic and hemorrhagic stroke subtypes. In this study, we analyzed management and outcomes according to stroke type (ischemic stroke (IS) and intracerebral hemorrhage (ICH)). Transient ischemic attacks and subarachnoid hemorrhages were excluded from this study.

During the early phase of this study (1992-1998), strokes were confirmed mainly on the basis of clinical examinations by senior neurologists for non-hospitalized patients and using medical records for hospitalized patients.

\section{Procedures for Ascertaining Stroke Events}

The procedures for ascertaining stroke events have been described previously. ${ }^{15}$ Briefly, stroke events were reported using predefined procedures. First, local village physicians reported initial stroke events to the community hospital within $24 \mathrm{~h}$ of stroke onset. Then, community hospital physicians visited the patients' homes to obtain clinical information and perform the initial diagnosis, which included taking histories of previous diseases and symptoms within $72 \mathrm{~h}$ after stroke onset. Thereafter, physicians reported all of the initially confirmed stroke events, diagnosed using computed tomography (CT) and/or magnetic resonance imaging (MRI), to TMUGH on a monthly basis. Suspected stroke events (ie, those with signs and symptoms of stroke but without confirmatory CT/MRI findings) were reported in real time. Finally, neurologists from TMUGH identified suspected cases via door-to-door interviews as soon as possible. Patients with a first-ever stroke and those with recurrent stroke were ascertained using the same procedure.

\section{Definition of Stroke Management and Outcomes}

Stroke management is based on the rates of strokes diagnosed by neuroimaging and of hospitalization. The rate of neuroimaging was defined as the proportion of patients who were diagnosed by CT or MRI from among all stroke patients. The hospitalization rate was defined as the proportion of inpatients from among all stroke patients. Stroke outcomes included the case fatality rate and the stroke recurrence rate. Case fatality was defined as the proportion of patients who died within 1 year after initial 
stroke among all stroke patients. Patients who died from other causes, including cancer, accident, and other nonstroke-related diseases, were excluded. The stroke recurrence rate was defined as the proportion of patients who had a recurrent stroke within 1 year of the first stroke occurrence among all stroke patients.

\section{Statistical Analysis}

Age is presented as mean (standard deviation [SD]), and the difference between two groups (sex and study period) was compared using the Student's $t$-test. Categorical variables are presented as number (\%), and the difference between two groups (sex and study period) was compared using the chi-squared test. The burden of stroke was compared for three periods (1992-2000, 2001-2009, and 2010-2018) using the chi-squared test. Trends in the burden of stroke were assessed as annual percentage changes (APCs) using the following regression model: $\operatorname{Ln}\left(r_{t}\right)=a+$ $\mathrm{bt}$, where $\mathrm{Ln}$ denotes the natural logarithm and $\mathrm{t}$ is the year. The trend, $b$, was estimated using ordinary regression, and $100 \times \mathrm{b}$ represents the estimated APC in the burden of stroke (including rates of patients diagnosed by neuroimaging, rates of hospitalization, case fatality, and recurrence). All statistical analyses were performed using
SPSS for Windows (version 15.0; SPSS, Chicago, IL, USA); a P-value $<0.05$ was considered statistically significant.

\section{Results}

\section{Demographic Characteristics for All Stroke Patients During the Study Period}

Overall, 1462 stroke cases occurred from 1992 to 2018, and $58.7 \%(\mathrm{n}=858)$ patients were male. The average age at stroke onset was 65.85 years; the mean age at the first-ever stroke was significantly younger in 2010-2018 than in 1992-2000. Although $38.7 \%$ of stroke patients had no formal education, the percentage of patients who were formally educated in primary school increased noticeably from $4.5 \%$ in $1992-2000$ to $27.1 \%$ in $2010-2018$. Moreover, ischemic stroke accounted for $77.6 \%$ of cases, with an increasing tendency (Table 1).

\section{Stroke Management and Outcomes}

Overall, the rates of patients diagnosed by neuroimaging and of hospitalization increased significantly over time in each of the three study periods: 1992-2000, 2001-2009, and 2010-2018. Similar trends were found across sexes, stroke types, and age groups. Case fatality within 1 year of

Table I Demographical Characteristics of All Patients by Study Periods

\begin{tabular}{|c|c|c|c|c|}
\hline Category & $1992-2000$ & $200 I-2009$ & $2010-2018$ & Overall \\
\hline Case, n (\%) & $245(16.8)$ & $509(34.8)$ & $708(48.4)$ & $1462(100.0)$ \\
\hline \multicolumn{5}{|l|}{ Gender: } \\
\hline Men & $153(62.4)$ & $294(57.8)$ & $4 I I(58.1)$ & $858(58.7)$ \\
\hline Women & $92(37.6)$ & $215(42.2)$ & $297(4 \mid .9)$ & $604(4 \mid .3)$ \\
\hline Age, years, means (SD) & 65.93 (1 I.39) & $66.87(11.98)$ & $65.08(11.96)$ & $65.85(11.89)$ \\
\hline \multicolumn{5}{|l|}{ Age group, $n(\%)$ : } \\
\hline$<45$ & $15(6.1)$ & $20(3.9)$ & $30(4.2)$ & $65(4.4)$ \\
\hline $45 \sim$ & $79(32.2)$ & $194(38.1)$ & $346(48.9)$ & $619(42.3)$ \\
\hline$\geq 65$ & $15 \mid(6 \mid .6)$ & $295(58.0)$ & $332(46.9)$ & $778(53.2)$ \\
\hline Education, years, means (SE) & $1.36(0.16)$ & $2.77(0.14)$ & $4.76(0.13)$ & $3.50(0.09)$ \\
\hline \multicolumn{5}{|l|}{ Education group, $\mathrm{n}(\%)$ : } \\
\hline 0 year & I72 (70.2) & $231(45.4)$ & $163(23.0)$ & $566(38.7)$ \\
\hline $\mathrm{I} \sim 6$ years & $62(25.3)$ & $228(44.8)$ & $353(49.9)$ & $643(44.0)$ \\
\hline$>6$ years & II (4.5) & $50(9.8)$ & $192(27.1)$ & $253(17.3)$ \\
\hline \multicolumn{5}{|l|}{ Stroke subtypes, n (\%): } \\
\hline Ischemic stroke & $176(7 \mid .8)$ & $379(74.5)$ & $580(81.9)$ & $1135(77.6)$ \\
\hline Hemorrhagic stroke & $51(20.8)$ & $104(20.4)$ & $116(16.4)$ & $27 \mid(18.5)$ \\
\hline Undefined & $18(7.3)$ & $26(5.1)$ & $12(1.7)$ & $56(3.8)$ \\
\hline
\end{tabular}

Abbreviations: SD, standard deviation; SE, standard error. 
initial stroke decreased remarkably over time, with a decline of $34 \%$ overall, $35.6 \%$ for men, and by $27.0 \%$ for patients aged 65 years and older. However, the recurrence rate remained stable during the three periods across all sex, age, and stroke types (Table 2).

\section{APC in Stroke Burden}

Table 3 shows the rate of diagnosis by neuroimaging increased significantly across all sex, age, and stroke type groups, with annual increases of $4.0 \%$ overall and of $5.2 \%$ for those aged $\geq 65$ years (all $\mathrm{P}<0.001$ ). The hospitalization rate increased remarkably over the three periods for all sex, age, and stroke type groups, with an overall annual increase of $11.2 \%$. The greatest increase was observed among patients aged $\geq 65$ years, who had an increase of $15.4 \%$. There was a noticeable decline in case fatality across sex, age, and stroke type (except ICH) groups over the past decade, with an overall decline of $3.5 \%$. The greatest decline was observed in women, who had an annual decrease of $4.4 \%$ (all $\mathrm{P}<0.05$ ). The

Table 2 Burden of Stroke During 1992 to 2018 by Gender and Stroke Types

\begin{tabular}{|c|c|c|c|c|}
\hline Category & $1992-2000$ & $200 I-2009$ & $2010-2018$ & $\mathbf{P}$ \\
\hline \multicolumn{5}{|l|}{ Total: } \\
\hline Diagnosis by $\mathrm{CT}$ & II $8(5 \mathrm{I} .8)$ & 381 (74.9) & $64 I(90.5)$ & $<0.001$ \\
\hline Hospitalized & $17(6.9)$ & $82(16.1)$ & $384(54.2)$ & $<0.001$ \\
\hline Case Fatality & $67(27.3)$ & $105(20.6)$ & 127 (I7.9) & 0.002 \\
\hline Recurrence & $19(7.8)$ & $49(9.6)$ & $67(9.5)$ & 0.523 \\
\hline \multicolumn{5}{|l|}{ Men: } \\
\hline Diagnosis by $\mathrm{CT}$ & $75(49.0)$ & 229 (77.9) & $368(89.5)$ & $<0.001$ \\
\hline Hospitalized & $10(6.5)$ & $41(13.9)$ & $229(55.7)$ & $<0.001$ \\
\hline Case Fatality & $43(28.1)$ & $63(21.4)$ & 77 (18.7) & 0.020 \\
\hline Recurrence & $14(9.2)$ & $33(11.2)$ & $44(10.7)$ & 0.698 \\
\hline \multicolumn{5}{|l|}{ Women: } \\
\hline Diagnosis by CT & $52(56.5)$ & $152(70.7)$ & 273 (91.9) & $<0.001$ \\
\hline Hospitalized & $7(7.6)$ & 41 (19.1) & $155(52.2)$ & $<0.001$ \\
\hline Case Fatality & $24(26.1)$ & $42(19.5)$ & $50(16.8)$ & 0.058 \\
\hline Recurrence & $5(5.4)$ & $16(7.4)$ & $23(7.7)$ & 0.510 \\
\hline \multicolumn{5}{|l|}{ IS: } \\
\hline Diagnosis by CT & $86(48.9)$ & $28 I(74.1)$ & $529(91.2)$ & $<0.001$ \\
\hline Hospitalized & $10(5.7)$ & $53(14.0)$ & $305(52.6)$ & $<0.001$ \\
\hline Case Fatality & $25(14.2)$ & $36(9.5)$ & $67(11.6)$ & 0.649 \\
\hline Recurrence & $18(10.2)$ & $40(10.6)$ & $59(10.2)$ & 0.932 \\
\hline \multicolumn{5}{|l|}{$\mathrm{ICH}:$} \\
\hline Diagnosis by $\mathrm{CT}$ & $41(80.4)$ & $100(96.2)$ & $112(96.6)$ & 0.001 \\
\hline Hospitalized & $7(13.7)$ & $29(27.9)$ & $79(68.1)$ & $<0.001$ \\
\hline Case Fatality & $25(49.0)$ & $44(42.3)$ & $48(4 \mid .4) 0.649$ & 0.407 \\
\hline Recurrence & I (2.0) & $9(8.7)$ & $8(6.9)$ & 0.382 \\
\hline \multicolumn{5}{|l|}{ < 65 years: } \\
\hline Diagnosis by $\mathrm{CT}$ & $72(76.6)$ & $197(92.1)$ & 372 (98.9) & $<0.001$ \\
\hline Hospitalized & $15(16.0)$ & $54(25.2)$ & $226(60.1)$ & $<0.001$ \\
\hline Case Fatality & II (II.7) & $20(9.3)$ & $37(9.8)$ & 0.718 \\
\hline Recurrence & 0 & $12(5.6)$ & $21(5.6)$ & 0.067 \\
\hline \multicolumn{5}{|l|}{$\geq 65$ years: } \\
\hline Diagnosis by $\mathrm{CT}$ & $55(36.4)$ & I84 (62.4) & $269(81.0)$ & $<0.001$ \\
\hline Hospitalized & $2(1.3)$ & $28(9.5)$ & I58 (47.6) & $<0.001$ \\
\hline Case Fatality & $56(37.1)$ & $85(28.8)$ & $90(27.1)$ & 0.040 \\
\hline Recurrence & $19(12.6)$ & 37 (12.5) & 46 (13.9) & 0.645 \\
\hline
\end{tabular}

Abbreviation: $\mathrm{CT}$, computed tomography. 
Table 3 APC of Stroke Burden During 1992 to 2018 by Gender and Stroke Types

\begin{tabular}{|c|c|c|c|c|c|c|c|c|}
\hline \multirow[t]{2}{*}{ Category } & \multicolumn{2}{|c|}{ Diagnosis by CT } & \multicolumn{2}{|c|}{ Hospitalized } & \multicolumn{2}{|c|}{ Case Fatality } & \multicolumn{2}{|c|}{ Recurrence } \\
\hline & APC $(95 \% \mathrm{Cl})$ & $\mathbf{P}$ & APC $(95 \% \mathrm{Cl})$ & $\mathbf{P}$ & APC $(95 \% \mathrm{Cl})$ & $\mathbf{P}$ & APC $(95 \% \mathrm{Cl})$ & $\mathbf{P}$ \\
\hline Total & $4.0(2.7,5.3)$ & $<0.001$ & II.2(9.3, I3.I) & $<0.001$ & $-3.5(-5.7,-1.4)$ & 0.003 & $-1.7(-4 . I, 0.7)$ & 0.150 \\
\hline \multicolumn{9}{|l|}{ Gender: } \\
\hline Men & $4.0(2.6,5.3)$ & $<0.001$ & $10.2(7.2,13.2)$ & $<0.001$ & $-3.5(-6.1,-0.9)$ & 0.012 & $-2.0(-4.4,0.5)$ & 0.106 \\
\hline Women & 4.I $(2.4,5.9)$ & $<0.001$ & $9.5(6.2,12.8)$ & $<0.001$ & $-4.4(-7.5,-1.3)$ & 0.008 & $-4.1(-7.4,-0.8)$ & 0.018 \\
\hline \multicolumn{9}{|l|}{ Age group } \\
\hline$<65$ years & $2.2(1.3,3.1)$ & $<0.001$ & $6.8(4.8,8.7)$ & $<0.001$ & $-3.7(-6.8,-0.6)$ & 0.024 & $-5.3(-11.1,0.6)$ & 0.074 \\
\hline$\geq 65$ years & $5.2(3.5,6.9)$ & $<0.001$ & $15.4(11.9,19.0)$ & $<0.001$ & $-3.1(-5.3,-0.9)$ & 0.009 & $-1.4(-3.6,0.8)$ & 0.191 \\
\hline \multicolumn{9}{|l|}{ Stroke types: } \\
\hline IS & $5.0(3.0,6.9)$ & $<0.001$ & II.8 (9.3, 14.4) & $<0.001$ & $-3.6(-6.3,-0.9)$ & 0.012 & $-3.6(-5.9,-1.2)$ & 0.005 \\
\hline $\mathrm{ICH}$ & $1.5(0.7,2.3)$ & 0.001 & $5.0(0.7,9.3)$ & 0.026 & $-1.0(-3.5,1.4)$ & 0.383 & $-1.3(-5.8,3.2)$ & 0.521 \\
\hline
\end{tabular}

Abbreviations: CT, computed tomography; APC, annual proportion of change; $\mathrm{Cl}$, confidence interval; IS, ischemic stroke; ICH, intracerebral hemorrhage.

recurrence rate significantly decreased by $4.1 \%$ for women and by $3.6 \%$ for the IS during the study period.

\section{APC by Sex, Age, and Stroke Type}

The rates of diagnosis by neuroimaging and of hospitalization increased noticeably across sex and age for all stroke types and particularly for IS, with the most obvious increases for men and women $\geq 65$ years of age (all $\mathrm{P}<0.05)$. The greatest increases were observed among elderly women, who showed a $4.7 \%$ increase in the rate of diagnosis by neuroimaging and a $12.4 \%$ increase in the rate of hospitalization (all $\mathrm{P}<0.001$ ). Case fatality from stroke decreased by $3.7 \%$ annually among those aged $<65$ years and by $3.1 \%$ among the elderly (aged $\geq 65$ years); the corresponding rates were $7.4 \%$ and $3.5 \%$ among women specifically (all $\mathrm{P}<0.05$ ). However, there was no significant improvement among men aged $<65$ years. The recurrence rate of IS significantly decreased among men, by $9.6 \%(\mathrm{P}=0.044)$ for those aged $<65$ years and by $3.1 \%(\mathrm{P}=0.033)$ for those aged $\geq 65$ years, with a decrease of $4.3 \%(\mathrm{P}=0.002)$ among women aged $\geq 65$ years (Table 4).

Table 4 APC of First-Ever Stroke Burden by Gender, Age, and Stroke Types During 1992 to 2018

\begin{tabular}{|c|c|c|c|c|c|c|}
\hline \multirow[t]{2}{*}{ Category } & \multicolumn{2}{|c|}{ Total } & \multicolumn{2}{|c|}{ Men } & \multicolumn{2}{|c|}{ Women } \\
\hline & $<65$ Years & $\geq 65$ Years & $<65$ Years & $\geq 65$ Years & $<65$ Years & $\geq 65$ Years \\
\hline \multicolumn{7}{|l|}{ Stroke, n (\%): } \\
\hline Diagnosis by CT & $2.2(1.3,3.1)^{*}$ & $5.2(3.5,6.9)^{*}$ & $1.7(0.3,3.0)^{*}$ & $4.7(2.9,6.4)^{*}$ & $1.6(0.7,2.4)^{*}$ & $4.7(2.4,7.0)^{*}$ \\
\hline Hospitalized & $6.8(4.8,8.7)^{*}$ & $15.4(11.9,19.0)^{*}$ & $5.2(2.3,8.0)^{*}$ & II.7 (7.8, I5.6)* & $5.5(2.2,8.7)^{*}$ & $12.4(8.0,16.7)^{*}$ \\
\hline Case Fatality & $-3.7(-6.8,-0.6)^{*}$ & $-3.1(-5.3,-0.9)^{*}$ & $-3.2(-8.5,2.1)$ & $-2.7(-4.9,-0.4)^{*}$ & $-7.4(-10.0,-4.7)^{*}$ & $-3.5(-6.8,-0.1)^{*}$ \\
\hline Recurrence & $-5.3(-1 \mathrm{I} . \mathrm{I}, 0.6)$ & $-1.4(-3.6,0.8)$ & $-7.7(-14.1,-1.3)^{*}$ & $-1.2(-3.6,1.1)$ & $-0.2(-8.3,8.0)$ & $-3.8(-6.7,-1.0)^{*}$ \\
\hline \multicolumn{7}{|l|}{ IS, n (\%): } \\
\hline Diagnosis by CT & $2.1(1.1,3.2)^{*}$ & $5.1(3.6,6.7)^{*}$ & $1.8(0.4,3.2)^{*}$ & $4.6(2.6,6.5)^{*}$ & $0.4(-0.3,1.1)$ & $4.4(2.1,6.8)^{*}$ \\
\hline Hospitalized & $6.8(4.2,9.4)^{*}$ & $13.9(9.1,18.8)^{*}$ & $5.1(1.3,9.0)^{*}$ & $10.8(5.0,16.7)^{*}$ & $2.5(-1.6,6.7)$ & $13.5(5.6,21.3)^{*}$ \\
\hline Case Fatality & $-2.3(-6.5,1.8)$ & $-2.7(-5.2,-0.2)^{*}$ & $-1.6(-39.7,36.5)$ & $-2.5(-6.6,1.6)$ & - & $-2.8(-6.4,0.7)$ \\
\hline Recurrence & $-7.3(-14.6,0.1)$ & $-3.1(-5.2,-0.9)^{*}$ & $-9.6(-18.8,-0.3)^{*}$ & $-3.1(-5.9,-0.3)^{*}$ & $-1.5(-6.5,9.5)$ & $-4.3(-6.7,-1.8)^{*}$ \\
\hline \multicolumn{7}{|l|}{$\mathrm{ICH}, \mathrm{n}(\%):$} \\
\hline Diagnosis by $\mathrm{CT}$ & $0.6(-0.1,1.3)$ & $0.8(-0.3,1.9)$ & $0.0(-0.2,0.1)$ & $0.7(-0.8,2.2)$ & $0.3(-0.2,0.8)$ & $0.4(-0.8,1.0)$ \\
\hline Hospitalized & $2.9(0.2,5.6)^{*}$ & $6.0(0.5,11.4)^{*}$ & $1.2(-2.8,5.2)$ & $0.5(-4.5,5.6)$ & $2.1(-1.5,5.8)$ & $3.4(-1.7,8.4)$ \\
\hline Case Fatality & $-2.1(-5.7,1.6)$ & $-0.7(-3.2,1.8)$ & $-1.2(-7.1,4.8)$ & $-0.4(-3.9,3.2)$ & $-2.7(-5.4,0.1)$ & $-1.7(-3.9,0.5)$ \\
\hline Recurrence & I.4 (-10.8, I3.5) & $2.7(-8.6,14.0)$ & $3.6(-25.1,32.3)$ & $12.9(-86.4,100)$ & $-20.9(-37.3,-4.4)^{*}$ & $-3.1(-13.5,7.2)$ \\
\hline
\end{tabular}

Note: ${ }^{*} \mathrm{P}<0.05$ compared between 1992-2008 and 2009-2018.

Abbreviations: $\mathrm{CT}$, computed tomography; IS, ischemic stroke; $\mathrm{ICH}$, intracerebral hemorrhage. 
In addition, the prevalence of hypertension, diabetes, smoking and alcohol in stroke patients showed in the Supplemental Table 1 . The prevalence of diabetes increased during the three study stages, from $2.5 \%$ during $1992-2000,10.5 \%$ during $2001-2009$, to $17.8 \%$ during 2010-2018 $(\mathrm{P}<0.001)$. Inversely, the rate of smoking decreased $(\mathrm{P}=0.050)$.

\section{Discussion}

This is the first report on long-term trends related to stroke management and burden in a low-income population in China. Since 1992, the rates of patients diagnosed by neuroimaging and of those hospitalized for stroke have increased substantially across sex, age, and stroke types; elderly women were observed to have the greatest increases. Moreover, there was a significant decrease in case fatality among women, with a decline of $7.4 \%$ among women aged $<65$ years and of $3.5 \%$ among women aged $\geq 65$ years. However, the recurrence rate of IS did not improve remarkably in women aged $<65$ years old.

Neuroimaging is the most important approach for distinguishing stroke subtypes, evaluating vascular patency and tissue vitality, and assessing the patient's eligibility for treatment as well as functional outcome. ${ }^{21,22}$ Furthermore, immediate CT scanning for acute stroke can reduce costs and improve quality of life. ${ }^{23} \mathrm{CT}$ was widely used in Chinese hospitals, in which approximately $90 \%$ of patients with IS underwent brain $\mathrm{CT}^{5}$ and almost $50 \%$ of patients underwent MRI. ${ }^{24}$ In this low-income population in China, we found that the rate of patients diagnosed by neuroimaging has increased substantially across sexes, ages, and stroke types since 1992, and elderly women had the greatest increase. The rate of patients diagnosed by neuroimaging was only $51.8 \%$ in the time period of 1992 to 2000 , whereas for 2010 to $2018,90.5 \%$ of stroke patients were diagnosed through neuroimaging. The rate of patients diagnosed by neuroimaging increased by $4.0 \%$ annually. The upward trend in the use of neuroimaging from 1992 to 2018 indicates that the level of medical care improved markedly in this low-income population in China following the national healthcare reform.

Between 2008 and 2014, 50.5\% of overall stroke patients were hospitalized in a stroke unit or intensive care unit (the rates by subtype were $51.1 \%$ for IS and $52.0 \%$ for ICH); and the average annual crude rates of IS patients hospitalized had increased for men and women aged 65 to 74 years but had decreased for those aged 75 to 84 years, while the opposite trend was observed for hemorrhagic stroke patients. ${ }^{25}$ Moreover, the age-specific acute IS hospitalization rates two national studies in the United States increased for individuals aged 15 to 44 years from 1995 to $2008^{26}$ and for those aged 25 to 65 years from 2000 to $2010 .^{27}$ A retrospective study performed in China showed that being hospitalized at an early stage of stroke was essential for recovery. ${ }^{28}$ However, prior to 2000 , only $6.9 \%$ of stroke patients were hospitalized in this low-income population, demonstrating the poor medical care they previously received. The rate of hospitalization for stroke increased to $54.2 \%$ during 2010 to 2018; the rates of hospitalization increased substantially across sex, age, and stroke types. More importantly, the greatest increase in the annual hospitalization rate for stroke was observed among women aged 65 years and older (12.4\%), suggesting that the healthcare reform in China vastly improved the number of women seeking medical advice, who are often affected by traditional culture and the lower social status for women. Changes in hospitalization rates reflected, to some extent, the improvement in stroke management and diagnosis in this low-income population, and in particular that the healthcare reform beginning in 2009 facilitated improvement in healthcare services for elderly women. Improved medical imaging, better knowledge of stroke symptoms among physicians and the overall population, and the deployment of stroke wards across the country may have improved the accuracy and speed of diagnosis and increased the rate of hospitalization. ${ }^{27}$ Furthermore, the improvement in the reformed healthcare system and in income improved the ability of rural residents to pay for medical care.

The age-standardized rate of stroke mortality had decreased by $33.4 \%$ overall from 1990 to 2017 and the mortality rate had decreased for all regions of varying socioeconomic status. ${ }^{29}$ Stroke mortality rates decreased by $55 \%$ from 2001 to 2010 , and the mean annual mortality change was $-6.0 \%$ for men and $-6.1 \%$ for women. ${ }^{30}$ Similar trends were found in 7 Western European countries. ${ }^{31}$ Consistent with this global trend, there was a $33.5 \%$ decrease in the age-standardized stroke mortality from 1990 to 2017 in China. ${ }^{32}$ However, mortality from stroke in rural areas remained relatively stable from 2003 to 2015 , and was consistently higher than that in urban areas. ${ }^{1}$ In addition to stroke, the mortality rate of lung cancer and CHD disease in the region is also showing an upward trend. ${ }^{33,34}$ From 1999 to 2016, the crude lung cancer death rate increased by $58.5 \%$, and the average annual change percentage (AACP) was $2.9 \%$. However, the age-standard YLL rate in 2016 was $13.3 \%$ lower than that in 1999, and the AACP was $-0.8 \%{ }^{33}$ From 1999 to 
2008, the age-standardized CHD mortality rate in Tianjin increased slightly, but the CHD mortality rate in various countries in the Americas and Europe showed a downward trend. ${ }^{34}$ For patients with first-ever stroke, appropriate and sufficient secondary prevention strategies have the potential to reduce the burden of stroke by up to one-quarter. $^{35}$ Compared with the untreated group, patients treated with anticoagulants and antiplatelets had reduced risks of death and recurrence from ischemic stroke (by $73 \%$ and $75 \%$, respectively). ${ }^{36}$ However, the use of secondary prevention medications for cardiovascular disease (ie, coronary heart disease or stroke) is low worldwide (antiplatelet drugs, 25.3\%; $\beta$ blockers, 17.4\%; angiotensin-converting enzyme inhibitors or angiotensinII receptor blockers, 19.5\%; and statins, 14.6\%), especially in low-income countries and rural areas. ${ }^{37}$ The use of antiplatelet and lipid-lowering treatments for secondary prevention has been shown to decline significantly after discharge from hospital in urban areas of China. ${ }^{38}$ The discontinuation of medications for secondary prevention can be attributed to socioeconomic factors, such as the price of the drugs and reimbursement policies, ${ }^{39}$ and poor compliance for long-term use drugs by patients and physicians. $^{38}$ In this study, the recurrence rate of stroke decreased remarkably annually among the elderly (by $3.1 \%$ for men and $4.3 \%$ for women) and among men aged $<65$ years (by 9.6\%). However, for women aged $<65$ years, this improvement was observed for ICH but not for IS. These findings suggest that there may be more emphasis on ICH than on IS in low-income populations; the heavy burden of stroke in rural areas, especially for young and middle-aged women, remained. In China, all residents aged 60 years and older receive free annual physical examinations supported by the government; moreover, patients with chronic NCD, including hypertension, diabetes, and cardiovascular disease, are interviewed door-to-door by community physicians to promote medical education and guide therapy. This practice may partly explain the better outcomes among elderly patients than among the younger and middle-aged patients in this study.

There are several limitations of this study. First, the study population was not a nationally representative sample, as study subjects were from one township in northern China. However, we assessed long-term trends for the burden of stroke in one low-income population in this study; therefore, the representativeness of the study population did not impact these results. Second, a limited number of stroke cases occurred during the study period. However, the long observation period was sufficient for the epidemiological study of stroke. Third, in this study's early observation period, the low rate of neuroimaging-based diagnoses may have affected the determination of stroke types and underrated stroke recurrence. Fourth, the effect of education stratification and blood pressure on stroke mortality is not accessed in this study, we will perform these stratified analysis futures. Finally, we did not collect the information of medication using, we will supplement it in the next study.

\section{Conclusion}

In conclusion, the management for stroke has improved substantially across sex, age, and stroke type groups over the past decades in Tianjin, China. The greatest improvement was observed among elderly women. Moreover, among elderly women, the case fatality rate decreased by $3.5 \%$ annually and the recurrence rate decreased by $3.8 \%$. However, the recurrence rate of IS in women aged $<65$ years did not improve significantly. These findings suggest that the allocation of medical resources has improved following economic development in China, and especially following healthcare reform that began in 2009. Women aged 65 years and older derived the greatest benefit from these improvements. However, it is crucial to enhance secondary stroke prevention by controlling risk factors in this low-income population, especially for women aged $<65$ years. At the same time, we should also pay attention to the risk of hypertension and cardiovascular disease in stroke patients to achieve early prevention. Moreover, to extend these benefits into rural China, it may be beneficial to implement integrated management into the care of patients with primary chronic NCD in low-income populations, such as by reducing the age at which free physical examination are available.

\section{Abbreviation}

TBS, The Tianjin Brain Study; TMUGH, Tianjin Medical University General Hospital; IS, Ischemic Stroke; ICH, Intracerebral Hemorrhage; CT, computed tomography; MRI, magnetic resonance imaging; SD, standard deviation; APCs, annual percentage changes; NCD, NonCommunicable Disease.

\section{Data Sharing Statement}

The datasets used and/or analysed during the current study are available from the corresponding author on reasonable request. 


\section{Ethics Approval and Informed Consent}

This study was conducted in accordance with the Declaration of Helsinki, and the study protocol was approved by the ethics committee of Tianjin Medical University General Hospital (TMUGH), and written informed consent was obtained from each participant (contract number: IRB2018-099-01).

\section{Acknowledgments}

We thank all participants of the Tianjin Brain Study and the local medical care professionals for their valuable contributions.

\section{Author Contributions}

All authors contributed to data analysis, drafting or revising the article, have agreed on the journal to which the article will be submitted, gave final approval of the version to be published, and agree to be accountable for all aspects of the work.

\section{Funding}

This research received no specific grant from any funding agency in the public, commercial or not-for-profit sectors.

\section{Disclosure}

The authors declare no conflicts of interest in this work.

\section{References}

1. Wu S, Wu B, Liu M, et al. Stroke in China: advances and challenges in epidemiology, prevention, and management. Lancet Neurol. 2019;18:394-405. doi:10.1016/S1474-4422(18)30500-3

2. Naghavi M, Abajobir AA, Abbafati Cet al. Global, regional, and national age-sex specific mortality for 264 causes of death, 19802016: a systematic analysis for the Global Burden of Disease Study 2016. Lancet. 2017;390:1151-1210. doi:10.1016/S0140-6736(17) 32152-9

3. Zhou M, Wang H, Zhu J, et al. Cause-specific mortality for 240 causes in China during 1990-2013: a systematic subnational analysis for the Global Burden of Disease Study 2013. Lancet. 2016;387:251-272. doi:10.1016/S0140-6736(15)00551-6

4. Feigin VL, Forouzanfar MH, Krishnamurthi R, et al. Global and regional burden of stroke during 1990-2010: findings from the Global Burden of Disease Study 2010. Lancet. 2014;383:245-254. doi:10.1016/S0140-6736(13)61953-4

5. Johnson CO, Nguyen M, Roth GA. Global, regional, and national burden of stroke, 1990-2016: a systematic analysis for the Global Burden of Disease Study 2016. Lancet Neurol. 2019;18:439-458. doi:10.1016/S1474-4422(19)30034-1

6. Liu M, Wu B, Wang WZ, Lee LM, Zhang SH, Kong LZ. Stroke in China: epidemiology, prevention, and management strategies. Lancet Neurol. 2007;6:456-464. doi:10.1016/S1474-4422(07)70004-2
7. Wang W, Jiang B, Sun H, et al. Prevalence, incidence, and mortality of stroke in China: results from a nationwide population-based survey of 480687 adults. Circulation. 2017;135:759-771. doi:10.1161/ CIRCULATIONAHA. 116.025250

8. Guan T, Ma J, Li M, et al. Rapid transitions in the epidemiology of stroke and its risk factors in China from 2002 to 2013. Neurology. 2017;89:53-61. doi:10.1212/WNL.0000000000004056

9. Wang Y, Xu J, Zhao X, et al. Association of hypertension with stroke recurrence depends on ischemic stroke subtype. Stroke. 2013;44:1232-1237. doi:10.1161/STROKEAHA.111.000302

10. Zhang N, Liu G, Zhang G, et al. External validation of the iScore for predicting ischemic stroke mortality in patients in China. Stroke. 2013;44:1924-1929. doi:10.1161/STROKEAHA.111.000172

11. Gu HQ, Li ZX, Zhao XQ, et al. Insurance status and 1-year outcomes of stroke and transient ischaemic attack: a registry-based cohort study in China. BMJ Open. 2018;8:e021334. doi:10.1136/bmjopen-2017021334

12. Li Z, Wang C, Zhao X, et al. Substantial progress yet significant opportunity for improvement in stroke care in China. Stroke. 2016;47:2843-2849. doi:10.1161/STROKEAHA.116.014143

13. Wang Y, Li Z, Zhao X, et al. Stroke care quality in China: substantial improvement, and a huge challenge and opportunity. Int J Stroke. 2017;12:229-235. doi:10.1177/1747493017694392

14. Wang Y, Li Z, Xian Y, et al. Rationale and design of a cluster-randomized multifaceted intervention trial to improve stroke care quality in China: the GOLDEN BRIDGE-acute ischemic stroke. Am Heart J. 2015;169:767-774.e2. doi:10.1016/j.ahj.2015. 03.008

15. Wang J, An Z, Li B, et al. Increasing stroke incidence and prevalence of risk factors in a low-income Chinese population. Neurology. 2015;84:374-381. doi:10.1212/WNL.0000000000001175

16. Wang J, Ning X, Yang L, et al. Sex differences in trends of incidence and mortality of first-ever stroke in rural Tianjin, China, from 1992 to 2012. Stroke. 2014;45:1626-1631. doi:10.1161/ STROKEAHA.113.003899

17. Wang J, Bai L, Shi M, et al. Trends in age of first-ever stroke following increased incidence and life expectancy in a low-income Chinese population. Stroke. 2016;47:929-935. doi:10.1161/ STROKEAHA.115.012466

18. Ning X, Sun J, Jiang R, et al. Increased stroke burdens among the low-income young and middle aged in rural China. Stroke. 2017;48:77-83. doi:10.1161/STROKEAHA.116.014897

19. Ministry of Health of the People's Republic of China. The causes of death, disease, and hurt in residents. China Health Statistics yearbook 2011. 2011:287-338.

20. Aho K, Harmsen P, Hatano S, Marquardsen J, Smirnov VE, Strasser T. Cerebrovascular disease in the community: results of a WHO collaborative study. Bull World Health Organ. 1980;58:113-130.

21. Zerna C, Thomalla G, Campbell B, Rha JH, Hill MD. Current practice and future directions in the diagnosis and acute treatment of ischaemic stroke. Lancet. 2018;392:1247-1256. doi:10.1016/ S0140-6736(18)31874-9

22. Hasan TF, Rabinstein AA, Middlebrooks EH, et al. Diagnosis and management of acute ischemic stroke. Mayo Clin Proc. 2018;93:523-538. doi:10.1016/j.mayocp.2018.02.013

23. Wardlaw JM, Seymour J, Cairns J, Keir S, Lewis S, Sandercock P. Immediate computed tomography scanning of acute stroke is cost-effective and improves quality of life. Stroke. 2004;35:2477-2483. doi:10.1161/01.STR.0000143453.78005.44

24. Huang Y, Wang JG, Wei JW, et al. Age and gender variations in the management of ischaemic stroke in China. Int $J$ Stroke. 2010;5:351-359. doi:10.1111/j.1747-4949.2010.00460.x 
25. Lecoffre C, de Peretti C, Gabet A, et al. National trends in patients hospitalized for stroke and stroke mortality in France, 2008 to 2014. Stroke. 2017;48:2939-2945. doi:10.1161/STROKEAHA.117.017640

26. George MG, Tong X, Kuklina EV, Labarthe DR. Trends in stroke hospitalizations and associated risk factors among children and young adults, 1995-2008. Ann Neurol. 2011;70:713-721. doi:10.1002/ ana. 22539

27. Ramirez L, Kim-Tenser MA, Sanossian N, et al. Trends in acute ischemic stroke hospitalizations in the United States. J Am Heart Assoc. 2016;5:e003233. doi:10.1161/JAHA.116.003233

28. Yu F, Liu X, Yang Q, Fu Y, Fan D. In-hospital recurrence in a Chinese large cohort with acute ischemic stroke. Sci Rep. 2019;9:14945. doi:10.1038/s41598-019-51277-8

29. Avan A, Digaleh H, Di Napoli M, et al. Socioeconomic status and stroke incidence, prevalence, mortality, and worldwide burden: an ecological analysis from the Global Burden of Disease Study 2017. BMC Med. 2019;17:191. doi:10.1186/s12916-019-1397-3

30. Seminog OO, Scarborough P, Wright FL, Rayner M, Goldacre MJ. Determinants of the decline in mortality from acute stroke in England: linked national database study of 795869 adults. BMJ. 2019;365:11778. doi:10.1136/bmj.11778

31. Kunst AE, Amiri M, Janssen F. The decline in stroke mortality: exploration of future trends in 7 Western European countries. Stroke. 2011;42:2126-2130. doi:10.1161/STROKEAHA.110.599712

32. Zhou M, Wang H, Zeng X, et al. Mortality, morbidity, and risk factors in China and its provinces, 1990-2017: a systematic analysis for the Global Burden of Disease Study 2017. Lancet. 2019;394:1145-1158. doi:10.1016/S0140-6736(19)30427-1

33. Zheng W, Zhang H, Shen C, et al. Trend analysis of lung cancer mortality and years of life lost (YLL) rate from 1999 to 2016 in Tianjin, China: does the lung cancer burden in rural areas exceed that of urban areas? Thorac Cancer. 2020;11(4):867-874. doi:10.1111/ 1759-7714.13314
34. Jiang G, Wang D, Li W, et al. Coronary heart disease mortality in China: age, gender, and urban-rural gaps during epidemiological transition. Rev Panam Salud Publica. 2012;31:317-324. doi:10.1590/S1020-49892012000400008

35. Hankey GJ. Secondary stroke prevention. Lancet Neurol. 2014;13:178-194. doi:10.1016/S1474-4422(13)70255-2

36. Faure M, Castilloux AM, Lillo-le-louet A, Bégaud B, Moride Y. Secondary stroke prevention: a population-based cohort study on anticoagulation and antiplatelet treatments, and the risk of death or recurrence. Clin Pharmacol Ther. 2020;107:443-451. doi:10.1002/ cpt. 1625

37. Yusuf S, Islam S, Chow CK, et al. Use of secondary prevention drugs for cardiovascular disease in the community in high-income, middle-income, and low-income countries (the PURE study): a prospective epidemiological survey. Lancet. 2011;378:1231-1243. doi:10.1016/S0140-6736(11)61215-4

38. Wei JW, Wang JG, Huang Y, et al. Secondary prevention of ischemic stroke in urban China. Stroke. 2010;41:967-974. doi:10.1161/ STROKEAHA.109.571463

39. Chen Y, Li L, Zhang Q, et al. Use of drug treatment for secondary prevention of cardiovascular disease in urban and rural communities of China: China Kadoorie Biobank Study of 0.5 million people. Int J Cardiol. 2014;172:88-95. doi:10.1016/j.ijcard.2013.12.065
Risk Management and Healthcare Policy

\section{Publish your work in this journal}

Risk Management and Healthcare Policy is an international, peerreviewed, open access journal focusing on all aspects of public health, policy, and preventative measures to promote good health and improve morbidity and mortality in the population. The journal welcomes submitted papers covering original research, basic science, clinical \& epidemiological studies, reviews and evaluations, guidelines, expert opinion and commentary, case reports and extended reports. The manuscript management system is completely online and includes a very quick and fair peer-review system, which is all easy to use. Visit http://www.dovepress.com/testimonials.php to read real quotes from published authors. 